\title{
PREVALÊNCIA de sINTOMAS RESPIRATÓRIOS EM SERVIDORES DE BIBLIOTECAS DE UMA UNIVERSIDADE PÚBLICA
}

\author{
Prevalence of respiratory symptoms in library \\ workers in a public university
}

Rogério de Souza Braga', Adilson Krumheuer de Azevedo², Lyandra Franco Carneiro ${ }^{3}$, Angela Luiza da Cunha Legey ${ }^{4}$, Arlete Ana Motter ${ }^{5}$

\footnotetext{
1. Universidade Federal do Paraná. ORCID: http://orcid.org/0000-0001-7707-1091

2. Universidade Federal do Paraná. ORCID: http://orcid.org/0000-0001-6152-5140

3. Universidade Federal do Paraná. ORCID: http://orcid.org/0000-0001-5549-5360

4. Universidade Federal do Paraná. ORCID: http://orcid.org/0000-0002-4295-4182

5. Universidade Federal do Paraná. ORCID: http://orcid.org/0000-0002-2585-207X

CONTATO: Arlete Ana Motter | Universidade Federal do Paraná (UFPR) | Setor de Ciências Biológicas - Curso de Fisioterapia | Avenida Cel. Francisco Heráclito dos Santos, s/n | Jardim das Américas | Curitiba | Paraná | Brasil CEP 81531-900 | Caixa Postal 19031 | E-mail: arlete.motter@uol.com.br
}

COMO CITAR Braga RS, Azevedo AK, Caneiro LF, Legey ALC, Motter AA. Prevalência de sintomas respiratórios em servidores de bibliotecas de uma universidade pública. R. Saúde Públ. 2018 Jul.;1(1):74-82.

RESUMO INTRODUÇÃO: Os servidores passam um terço do dia no ambiente de trabalho, podendo ser um fator desencadeante de doenças ocupacionais, destacando-se os problemas respiratórios. OBJETIVO: Descrever a prevalência de sintomas respiratórios em profissionais de bibliotecas de uma universidade pública do sul do Brasil. MATERIAIS E MÉTODOS: O projeto desenvolveu-se entre 2015 e 2016. A amostra conta com servidores de ambos os sexos, respondendo a dois instrumentos de coleta de dados: questionário respiratório e questionário de Fargestron para dependência ao tabagismo. RESULTADOS: Foram selecionadas 14 bibliotecas da instituição, 
136 trabalhadores, sendo 68\% mulheres, e 32\% homens. Destes, $44 \%$ relataram sintomas como, tosse com secreção (36\%), tosse seca (23\%), cansaço (19\%), chiado (9\%), dor no peito (5\%). Das patologias, bronquite (40\%), rinite (23\%), foram as mais relatadas. CONCLUSÃO: É necessária maior participação de profissionais da saúde em ambientes de trabalho como bibliotecas, visando promover aos servidores, saúde, segurança e educação no trabalho.

PALAVRAS-CHAVE: Doenças ocupacionais. Sinais e Sintomas Respiratórios. Bibliotecários.

\begin{abstract}
INTRODUCTION: Public servants spend a third of the day in the work environment, which can be a triggering factor for occupational diseases, especially respiratory problems. OBJECTIVE: To describe the prevalence of respiratory symptoms in professionals working in libraries of a public university in the south of Brazil. MATERIALS AND METHODS: The project was developed between 2015 and 2016. The sample has employees of both genders, responding to two instruments of data collection: respiratory questionnaire and Fargestron questionnaire for smoking dependence. RESULTS: Fourteen libraries of the institution were selected, with 136 workers, $68 \%$ female, and 32\% male. Of these, $44 \%$ reported symptoms such as cough with secretion (36\%), dry cough (23\%), fatigue (19\%), wheezing (9\%), chest pain (5\%). Of the pathologies, bronchitis (40\%) and rhinitis (23\%) were the most reported. CONCLUSION: There is a need for greater participation of health professionals in work environments such as libraries, aimed at promoting health, safety, and education at work.
\end{abstract}

KEYWORDS: Occupational diseases. Signs and Symptoms, Respiratory. Librarians.

\title{
INTRODUÇÃO
}

$\mathbf{A}$

dedicação ao trabalho hoje ocupa um terço do dia das pessoas. Tal índice é baseado na carga horária de trabalho de quarenta horas semanais com exercício de atividades durante oito horas por dia, cinco vezes na semana. Para que tais questões não afetem negativamente o desempenho profissional e social do indivíduo é necessário proporcionar uma boa qualidade de vida no trabalho.

A profissão de Biblioteconomia é regulamentada pela Lei $n^{\circ}$. 4.084 de 30 de junho de 1962 que dispõe sobre seu exercício o decreto n. 56.725 de 16 de agosto de 1965, que destaca em seu Artigo $2^{\circ}$ o profissional bibliotecário no quadro de profissões liberais da Consolidação das Leis de Trabalho (CLT). A promoção de capacitação e educação desses profissionais é função da Federação Brasileira de Associações de Bibliotecários, Cientistas da Informação e Instituições (FEBAB), fundada em julho de 1959, e a fiscalização é responsabilidade do Conselho Federal de Biblioteconomia (CFB), criado em dezembro de $1965^{2}$

O profissional bibliotecário tem como principal ponto de atuação a informação, com processos de geração, disseminação, recuperação, gerenciamento, conservação e utilização da informação. ${ }^{3}$ Essa atuação é bastante diversificada, com atuação em diferentes setores não só em bibliotecas, mas arquivos, centros de documentação, jornais, estações de televisão, internet, museus, livrarias e editoras, escritórios de advocacia e contabilidade e organização de bases de dados virtuais. ${ }^{4}$ 
As bibliotecas, arquivos e museus são edificações voltadas à preservação e com a finalidade de conservar materiais com a finalidade da durabilidade, possibilitando acesso ao conhecimento por meio das coleções de livros, documentos e objetos para a população em geral. Apresentando uma diversificada proporção de locais de trabalho para diferentes cargos neles exercidos como bibliotecários, curadores, arquivistas, conservadores entre outros. Esses locais de trabalho podem apresentar pouca ou nenhuma ventilação sendo um fator desencadeante de inalantes prejudicais à saúde desses trabalhadores, muitas vezes expostos sem nenhuma proteção impactando sua saúde, bem-estar e a produtividade. Nesses ambientes, a presença de microrganismos como bactérias, fungos e vírus podem ser encontrados em livros de diferentes materiais, no mobiliário como estantes, armários, arquivos de armazenamento com pouco ou nenhuma ventilação resultando no aumento da umidade e estimulando a surgimento da microbiota fúngica geralmente patogênica. ${ }^{5-6-7-8-9-10}$

As doenças ocupacionais são classificadas em fatores de risco, podendo ser físicos quando relacionados à climatização e ruídos; químicos quando agentes aos quais o profissional é diretamente exposto; biológicos por vírus, bactérias ou parasitas presentes ou relacionados ao ambiente de trabalho; ergonômicos e psicossociais relacionados à má organização e má gestão do trabalho, resultando em um ambiente inadequado

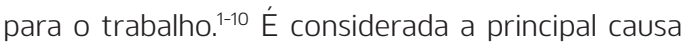
de incapacidade no trabalho, fator esse relacionado às horas internas em ambientes com contaminação por agentes irritativos e imunogênicos à saúde. $A$ asma é considerada uma doença respiratória de incapacidade no trabalho em sujeitos entre 18 a 44 anos. ${ }^{10-11-12}$

Outros problemas relacionados ao ambiente de trabalho são a contaminação do ar por poeira, comumente encontrada em ambientes com pouca ventilação e importante agente das doenças respiratórias, quando inaladas podem apresentar alergias respiratórias, micoses alérgicas broncopulmonares e pneumonias hipersensitivas. ${ }^{9}$
Existem diferentes tipos de trabalho, cada um com suas respectivas atividades desenvolvidas em inúmeros setores. Estes ambientes podem apresentar agentes inaláveis, por exemplo, a área da panificação, em que os trabalhadores podem ficar expostos à poeira da farinha de trigo, exposição que pode resultar em asma, rinite, além de outros sintomas respiratórios. ${ }^{13}$ No setor agrícola, os trabalhadores da produção de biocombustível e da queima da palha de canade-açúcar, como os da indústria de cerâmica, são sujeitos às altas temperaturas e à inalação de partículas de poeira como de fumaça, as quais muitas vezes são depositadas nas vias aéreas, desenvolvendo inúmeras doenças respiratórias. ${ }^{14-15}$ Não só a poluição do ar é um fator desencadeante de doenças respiratórias ocupacionais, pois outros fatores como, por exemplo, a Síndrome do Edifício Doente, são relacionados à contaminação de um determinado ambiente por uma errônea climatização da umidade e temperatura que favorece a proliferação de microrganismos responsáveis pela biodeterioração do edifício, materiais e outros objetos nele encontrados, além de causar alergias, infecções e intoxicação. ${ }^{16-17-18}$

A pesquisa de prevalência de sintomas respiratórios em bibliotecários pela abordagem da fisioterapia do trabalho e saúde do trabalhador tem como justificativa alertar e assim minimizar os agravos à saúde desses profissionais, na medida em que ações preventivas e educativas poderão ser tomadas e expandidas para outras situações similares de trabalho.

\section{MATERIAIS E MÉTODOS}

O estudo é do tipo transversal analítico e desenvolveu-se entre setembro de 2015 a julho de 2016 nas bibliotecas de uma universidade pública do sul do país. Com base no número total de funcionários do setor pesquisado ( $n=200)$, utilizouse uma amostra constituída por 136 funcionários, correspondente a uma margem de erro igual a 5\% e um intervalo de confiança de 95\%. 
Os critérios de inclusão de seleção dos participantes foram trabalhadores que desempenhavam funções nas bibliotecas e na área administrativa que concordaram em participar da pesquisa segundo o Termo de Consentimento Livre e Esclarecido.

Foram utilizados dois questionários como instrumento de coleta de dados: 1) Questionário respiratório e 2) Questionário de Fargeströn (dependência ao tabagismo). O questionário de Fargeströn foi inserido na investigação, pois, como se sabe, o tabagismo é considerado um fator de risco importante para sintomas e doenças respiratórias.

O Questionário de sintomas respiratórios contou com 53 questões (24 questões abertas e 29 questões fechadas), elaborado especificamente para esta investigação. As primeiras questões se refeririam à identificação do participante, seguido de seus hábitos de vida. Após, foram colocadas questões sobre características ambientais, organizacionais e das atividades realizadas na jornada de trabalho. Por fim, abordaram-se aspectos sobre a incidência de características desencadeantes de patologias respiratórias.

O questionário de Fargeströn é composto por 6 questões objetivas, tem o intuito de avaliar a dependência à nicotina. ${ }^{19}$ A partir dos dados coletados, quando preciso, foram calculados os anos-maços e adicionados à ficha de avaliação do participante. Desenvolvido em 1978 e validado no Brasil em 1991, onde, em cada alternativa, há uma pontuação e a soma disso resultará na avaliação do grau de dependência da nicotina.

Os questionários foram aplicados no próprio ambiente de trabalho dos indivíduos em dias distintos, tanto no período da manhã, quanto no período da tarde. Cada entrevista durou, em média, 15 minutos. Primeiramente o aluno se apresentava para a chefia de cada local a ser estudado. Com isto acabamos contando com a ajuda deste profissional para realizar o convite aos servidores e também nos cedendo uma sala mais reservada para a realização da entrevista de uma forma mais intimista. Após assinar o termo de consentimento, realizava-se a aplicação dos questionários onde o pesquisador enunciava as questões para evitar divergência de interpretação, e anotava as respostas relatadas pelo participante.20

Quanto à análise dos dados, em relação ao tabagismo, os participantes foram categorizados em tabagistas e não tabagistas. Quanto às atividades desenvolvidas nas bibliotecas, foram consideradas cinco categorias (chefe de biblioteca, bibliotecário, auxiliar de biblioteca, estagiário, assistente administrativo). Para avaliar o grau de exposição nas bibliotecas, utilizou-se o tempo de trabalho nas bibliotecas da universidade, em qualquer uma das cinco ocupações consideradas no presente estudo, sendo essa variável categorizada em até 5 anos e mais de 5 anos. Para classificar a jornada de trabalho do funcionário, a carga horária semanal foi dividida em quatro categorias: 20 horas semanais, 30 horas semanais, 40 horas semanais.

Quanto às variáveis dependentes, consideraram-se como "sintomas respiratórios gerais" aqueles trabalhadores que responderam apresentar um dos cinco sintomas respiratórios questionados (tosse, tosse com secreção, chiado, dor no peito, cansaço (falta de ar) e, como "sintomas respiratórios graves", aqueles trabalhadores que responderam apresentar uma das cinco variáveis de diagnóstico de doenças respiratórias (presença de doenças respiratórias, asma, bronquite alérgica, bronquite asmática, pneumonia, rinite).

O projeto de pesquisa foi aprovado pelo Comitê de Ética em Pesquisa da própria universidade sob o número 1175594 em 07 de agosto de 2015.

\section{RESULTADOS}

Foram selecionadas 14 bibliotecas da instituição de ensino, totalizando 136 trabalhadores de bibliotecas. Dos 136 trabalhadores, 93 (68\%) eram mulheres, e 43 (32\%) eram homens, com média de idade de 41,11 \pm 14,05 anos, média de horas trabalhadas por semana de $30 \pm 5,26 \mathrm{~h}$ e tempo nessa atividade 8,54 \pm 9,48 anos. A Tabela 1 mostra as características dos trabalhadores nas bibliotecas estudadas. 
Tabela 1. Características dos trabalhadores de bibliotecas do estudo.

\begin{tabular}{|c|c|}
\hline VARIÁVEIS & $\begin{array}{l}\text { TRABALHADORES } \\
(\mathrm{N}=136)\end{array}$ \\
\hline Sexo feminino ${ }^{a}$ & $93(68 \%)$ \\
\hline Sexo masculino ${ }^{a}$ & $43(32 \%)$ \\
\hline Idade b, anos & $41,11 \pm 14,05$ \\
\hline Hábito tabágico a & $17(12 \%)$ \\
\hline \multicolumn{2}{|c|}{ Dados ocupacionais } \\
\hline Horas trabalhadas/semana b & $30 \pm 5,26$ \\
\hline Anos de trabalho b & $8,54 \pm 9,48$ \\
\hline Uso de EPIs ${ }^{\mathrm{a}}$ & $52(40 \%)$ \\
\hline \multicolumn{2}{|c|}{ Tipo de atividade ${ }^{a}$} \\
\hline Chefia & $7(5 \%)$ \\
\hline Bibliotecário & $36(26 \%)$ \\
\hline Auxiliar de biblioteca & $35(26 \%)$ \\
\hline Estagiário & $23(17 \%)$ \\
\hline Administrativo & $35(26 \%)$ \\
\hline
\end{tabular}

EPI: equipamento de proteção individual. a Resultados expressos com $n$ (\%). b Resultados expresso com média $\pm d p$. Fonte: Elaborada pelos autores (2018)

A Tabela 2 apresenta a ocorrência dos sintomas respiratórios nos trabalhadores de bibliotecas, assim como as possiveis doenças relatadas. Dos 136 indivíduos entrevistados, a presença de tosse (23\%), tosse com secreção (36\%), chiado (9\%), dor no peito $(5 \%)$, cansaço $(19 \%)$ foram os sintomas mais frequentes relatados pelos trabalhadores. Quando perguntados sobre se já apresentaram ou apresentam doenças respiratórias, 44\% relataram que sim. E quando questionados sobre quais eram, asma, bronquite alérgica, bronquite asmática, pneumonia e rinite foram as doenças mais relatadas (14\%, 40\%, 19\%, 5\% e 23\% respectivamente).

Ao analisar a relação dos sintomas respiratórios apresentados pelos trabalhadores com hábito tabágico (Gráfico 1), foi possível observar que $71 \%$ dos trabalhadores tabagistas não apresentam ou apresentaram alguma doença respiratória, como 54\% dos trabalhadores não tabagistas também não apresentaram doenças respiratórias.

O Gráfico 2 compara o tempo na função no cargo segundo a presença de doenças respiratórias (DR).
Tabela 2. Sintomas respiratórios e diagnósticos de doenças respiratórias.

\begin{tabular}{lc}
\hline SINTOMAS & $\mathbf{n}(\%)$ \\
RESPIRATóRIOS & $31(23 \%)$ \\
Tosse & $12(36 \%)$ \\
Tosse com secreção & $12(9 \%)$ \\
Chiado & $7(5 \%)$ \\
Dor no peito & $25(19 \%)$ \\
Cansaço (falta de ar) & \\
& \\
Presença de DR & $59(44 \%)$ \\
Asma & $6(14 \%)$ \\
Bronquite alérgica & $17(40 \%)$ \\
Bronquite asmática & $8(19 \%)$ \\
Pneumonia & $2(5 \%)$ \\
Rinite & $10(23 \%)$ \\
\end{tabular}

$D R$ : doença respiratória

Fonte: Elaborada pelos autores (2018)

Gráfico 1. Relação dos trabalhadores Tabagistas e Não Tabagistas com presença de Doenças Respiratórias (DR).

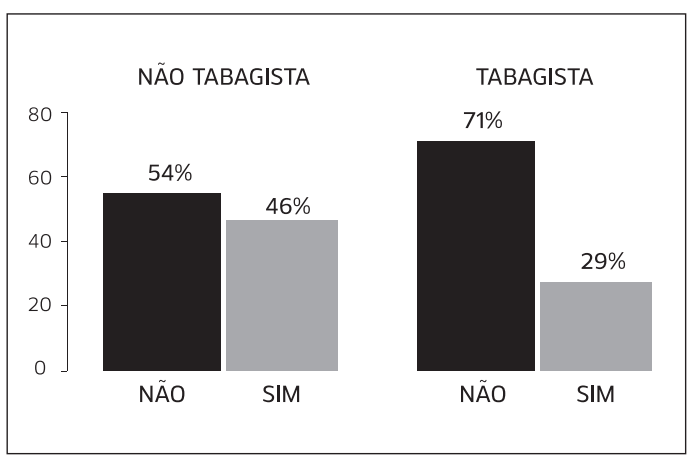

Fonte: Elaborada pelos autores (2018)

Gráfico 2. Relação do tempo na função com a presença de doenças respiratórias.

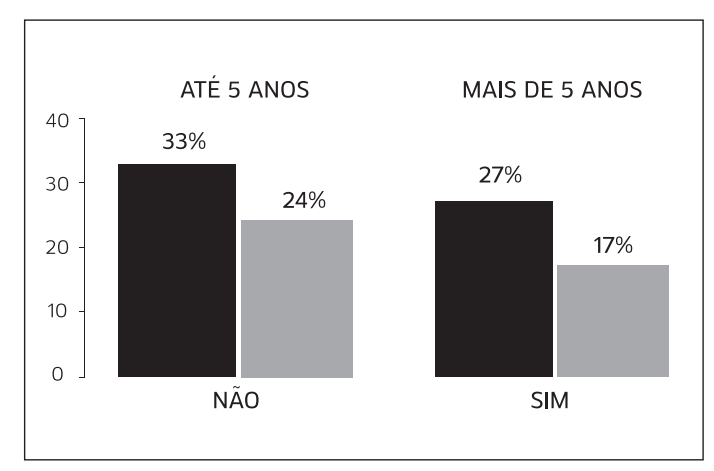

Fonte: Elaborada pelos autores (2018) 
Para os que estão na função até 5 anos, 33\% não apresentaram alguma DR, e para os indivíduos com mais de 5 anos foi de $24 \%$.

Dentre os que apresentam alguma doença respiratória, 27\% dos que exercem a função até 5 anos, enquanto os que exercem há mais de 5 anos é de $17 \%$.

Com isso nota-se que, para esses indivíduos, não houve uma diferença tão grande em relação aos anos na função exercida para a presença de DR.

O Gráfico 3 apresenta a relação do tempo na função exercida com a utilização de equipamentos de proteção individual (EPI). 40\% dos que estão na função até 5 anos não utilizam EPI e 21\% dos que trabalham há mais de 5 anos também não utilizam. Dentre os que utilizam, temos $20 \%$ com função exercida até 5 anos, enquanto os que trabalham há mais de 5 anos é de $21 \%$. Podemos concluir que os indivíduos até 5 anos na função não utilizam EPIs, tornando-se uma tendência a não utilizar conforme o passar dos anos, sendo um fator para a presença de DR.

Gráfico 3. Relação do tempo da função com a utilização de Equipamentos de Proteção Individual (EPI).

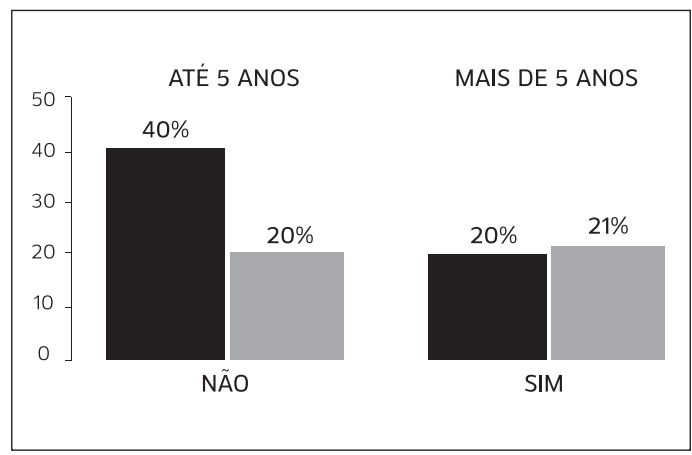

Fonte: Elaborada pelos autores (2018)

\section{DISCUSSÃO}

Com base nos resultados, foi possível verificar que as características do trabalho em bibliotecas podem contribuir para o desenvolvimento de sintomas ocupacionais. Tais sintomas podem ser desencadeados tanto pelo trabalho desenvolvido quanto por hábitos de vida do indivíduo, levando em consideração o comportamento de saúde desses indivíduos.

Existem inúmeras doenças relacionadas ao trabalho que afetam trabalhadores de diferentes ambientes e cada uma com seu respectivo sintoma. Ambientes com pouca ventilação como as bibliotecas, são fatores para os sintomas respiratórios, bronquite alérgica, asma, rinite, tosse. ${ }^{11}$ Esses resultados são semelhantes a outro estudo, onde a presença de sintomas respiratórios em trabalhadores de uma mineração foi relatada por $22,44 \%$, com relatos de mais um sintoma, com ressalto nos sintomáticos 35,08\% com história de tabagismo ou ex-tabagismo. ${ }^{19}$ Apesar de serem profissões distintas e ambientes totalmente diferentes, os sintomas se assemelham em ambos os trabalhadores. Estes dados também corroboram com outro estudo, onde 66,67\% de trinta bibliotecários entrevistados possuem algum tipo de doença ou alergia de ordem respiratória, sendo a rinite a mais prevalente em $55 \%{ }^{22}$

Outro estudo com funcionários de uma biblioteca pública apresenta resultados com sintomas semelhantes, nos quais houve queixas como garganta seca e irritada (29\%), espirros e coceira nasal (19\%) e olhos lacrimejantes (15\%). No entanto, esses resultados estão relacionados à Síndrome do Edifício Doente (SED), cujos sintomas regridem após o sujeito se afastar do ambiente em questão. ${ }^{23}$

Os sintomas respiratórios (presença de tosse. tosse seca, tosse com secreção, chiado, dor no peito, cansaço) dos participantes da pesquisa estão associados aos relatos de doenças respiratórias, tais como asma, bronquite alérgica, bronquite asmática, pneumonia e rinite. Merecem destaques a presença de tosse com secreção, como se vê na Tabela 2, e as doenças respiratórias de caráter alérgico. Esses resultados corroboram aos achados de um estudo realizado em carvoarias com 67 trabalhadores na produção de carvão vegetal, onde se observou a presença de espirros e secreção nasal em 35,82\% 
dos trabalhadores, já a tosse, expectoração e dispneia também foram relatadas em 22,38\%, $26,86 \%$ e $11,94 \%$ respectivamente.

No estudo de Setto et al., os indivíduos que apresentaram doenças respiratórias, como asma ocupacional, eram tabagistas, já a DPOC foi diagnosticada em 4 indivíduos, seguida de 3 indivíduos com diagnóstico de bronquite crõnica. ${ }^{24}$

Em estudo realizado com servidores públicos de uma universidade pública federal da região sudeste do Brasil, demonstrou-se que o uso de tabaco foi autorrelatado por $8,7 \%$ dos empregados, enquanto 91,3\% eram não fumantes. Em seus resultados, os autores afirmam não haver associação estatística com doenças respiratórias. Os resultados são similares aos observados neste estudo, onde apenas 12\% dos trabalhadores entrevistados têm hábito tabágico, mas que não apresentam resultados significativos para doenças respiratórias. ${ }^{25}$

Referindo-se à segurança no trabalho, foi questionada a utilização de equipamento de proteção individual (EPI), sendo observado que o hábito do uso de EPI é menor nos indivíduos com menos tempo na função, contrariando dados obtidos por outros autores. ${ }^{18}$ Em estudo também realizado com bibliotecários de Salvador. observou-se que $66,67 \%$ fazem uso dos EPIs e $33 \%$ não utilizam. Esse mesmo questionamento da não utilização de EPI corrobora com estudos, onde questionaram bibliotecários sobre o uso de EPI e 52\% responderam que não usavam ${ }^{19}$. Portanto, esse aspecto deverá ser repensado para o caso em questão, visto que uma má educação em relação à segurança no trabalho, como, por exemplo, na agricultura, onde o uso de EPIs não é visto com importância, torna-se fator contribuinte para doenças respiratórias relatadas pelos trabalhadores. ${ }^{26}$

Uma pesquisa realizada com funcionários de uma Instituição de Ensino Superior Estadual chegou à conclusão que o aprimoramento do nível de instrução do trabalhador é um aspecto fundamental para a qualidade de vida no ambiente de trabalho. ${ }^{23}$ Isso pode ser uma explicação pelo fato de haver poucos tabagistas e estes apresentarem poucos problemas de saúde relacionados ao trabalho. Além disso, também foi observado que os servidores trabalham em média 30h semanais, isso pode contribuir positivamente sobre a qualidade de vida profissional e particular, pois assim estes servidores dispõem de mais tempo para cuidados com a família, saúde, entre outros.

Pelos resultados pode-se constatar que o grupo de participantes com menos anos de trabalho na instituição apresentou-se com discreto aumento de doenças respiratórias, justamente o grupo no qual 40\% deles revelaram que não usam EPI (Gráfico 2).

Os tabagistas e não tabagistas apresentaram resultados muito similares quanto à presença de doença respiratória (Gráfico 1). Este resultado corrobora ao estudo, ${ }^{27}$ com servidores técnicoadministrativos de uma universidade pública, no qual o autor relacionou as doenças ao tabagismo. Resultando que $85 \%$ dos servidores não apresentaram doença relacionada ao tabagismo e $6 \%$ relataram já terem apresentado doença relacionada ao tabagismo. Dentre as doenças citadas pelos entrevistados estão as alergias respiratórias, bronquite/bronquite asmática, hipertensão, infarto e pneumonia.

A presença de doenças associadas ao tabagismo em trabalhadores de um hospital universitário foi relatada por 18,8\% dos trabalhadores ${ }^{28}$, sendo frequente em ex-fumantes $(p=0,008)$, dentre as principais doenças citadas foram circulatórias, 124 (8,4\%), respiratórias, 99 (6,7\%), psiquiátricas, 17 (1,1\%) e gastrointestinais, 17 $(1,1 \%)^{25}$

Qualquer ambiente de trabalho pode apresentar riscos à saúde do profissional que nele desempenha sua função. Por exemplo, Souza et $a^{23}$, identificaram em carvoarias 8,80 vezes mais prurido nasal ao desempenhar a função com forno aceso quando comparados aos trabalhadores não tabagistas. A obstrução nasal está aumentada em trabalhadores tabagistas e eles têm 5,00 vezes mais risco de apresentar tosse, além de 10,58 vezes maior chance de apresentar sibilância. Por isso, 
apesar deste estudo apresentar baixa correlação entre o tabagismo e as doenças respiratórias, tal hábito constitui um fator de risco respiratório ao qual se deve estar atento.

Um estudo na faculdade de medicina da Bahia realizou uma intervenção com o intuito de melhorar a qualidade do ar ambiente na biblioteca, já que havia uma grande diferença na quantidade de fungos no ambiente interno (204ufcm3) e no externo (117ufcm3). Esta intervenção se deu por neblina de água com surfactante e bioaerossóis, mostrando-se eficaz, diminuindo para 10,6 ufcm3 a quantidade de fungos no ambiente interno da biblioteca. ${ }^{29}$

\section{CONCLUSÃO}

O estudo permitiu identificar que o trabalho em biblioteca contribui para o desencadeamento de sintomas ocupacionais relacionado ao trabalho, o que pode interagir e interferir diretamente na qualidade de vida do trabalhador e em sua produtividade. Os cuidados com a saúde do trabalhador se fazem importantes para a promoção da qualidade de vida e consequentemente o melhor desempenho da atividade laboral. A escassez de estudos no Brasil demonstra a necessidade de realizar atividades de educação e conscientização como método de promoção e prevenção em saúde com embasamento científico. Assim sendo, são necessários mais estudos nesta área para que seja possivel identificar com mais detalhamento a correlação das características ambientais das bibliotecas e a saúde do trabalhador, promovendo um método baseado na evidência.

\section{AGRADECIMENTOS}

Agradecemos grandiosamente ao Prof. Dr. João Adriano de Barros, Pneumologista do Complexo Hospital de Clínicas da UFPR, pelas reuniões e discussões técnicas com profundo embasamento científico no qual foi possível obter um visão crítica sobre o assunto abordado na pesquisa.

\section{REFERÊNCIAS}

1. Araújo LS. A importância da ergonomia aplicada às unidades de informação para a atuação profissional dos bibliotecários. Rio de Janeiro: Trabalho de conclusão de curso, Escola de Biblioteconomia - Universidade Federal do Estado do Rio de Janeiro, Rio de Janeiro. 2014.

2. Spudeit DFAO, Fuhr F. Sindicatos de bibliotecários: história e atuação. Rev TransInformação. 2011; 23:235-249.

3. Duarte EA, Braga RMO. O profissional bibliotecário e o domínio da língua inglesa. R Eletr Bibliotecon. 2010; 30(15):105-122.

4. Amato GD, Cecchi L, Amato MD, Liccardi G. Urban air pollution and climate change as environmental risk factors of respiratory allergy: an update. J Investig Allergol Clin Immunol. 2010; 20(2):95-102.

5. Reis Menezes AA. Fungos em bibliotecas: frequência dos gêneros em livros e elaboração de teste para avaliação da biorreceptividade em papéis [dissertação]. São Paulo: Instituto de Biomédicas, Universidade de São Paulo. 2009.

6. Skora J, Gutarowska B, Pielech-Przybylska K, Stepién L, Pietrzak K, Piotrowska M, et al. Assessment of microbiological contamination in the work environments of museums, archives and libraries. J. Aerobiologia. 2015; 31: 389- 401.

7. Hayleeyesus SF, Manaye AM. Microbiological quality of indoor air in university libraries. Asian Pac J Trop Biomed. 2014; 4(1):312317.

8. Backes LTH, Naumann VLD, Calil LN. Isolamento de fungos anemófilos em biblioteca e prevalência de alergias respiratórias. Rev Panam Infectol. 2011; 13(3):19-25.

9. Ribeiro EL. Fungos na biodeterioração de livros em ambientes bibliotecários nos últimos 35 anos (1977- 2012). Revista Brasileira de Biblioteconomia e Documentação. 2013; 9(1):17-27.

10. Fernandes ALG, Stelmach R, Algranti E. Asma ocupacional. J Bras Pneumol. 2006; 32(1):27-34.

11. Branco ABA, Ildefonso SAG. Prevalência e duração dos benefícios auxílio-doença decorrentes de asma no Brasil em 2008. J Bras Pneumol. 2012; 38(5):550-558.

12. Galvão CES. Asma e rinite ocupacionais - visão imunoalérgica. Rev Bras alerg. imunopatol. 2010; 33(1):2-7.

13. Denipotti MEP, Robazzi MLCC. Riscos ocupacionais identificados nos ambientes de panificação brasileiros. Rev Cienc Enferm. Concepción. 2011; (1):117-127.

14. Ribeiro H. Queimadas de cana-de-açúcar no Brasil: efeitos à saúde respiratória. Rev Saúde Pública. 2008; 42(2):370-376.

15. Rondon EN, Silva RMVG, Botelho C. Sintomas respiratórios como indicadores de estado de saúde em trabalhadores de indústrias de cerâmicas. J Bras Pneumol. 2011; 37(1):36-45.

16. Toloza MDL, Lizarazo FLM, Blanco VJO. Concentración y composición microbiana em el ambiente d ela biblioteca centra Jorge palácios preciado de la universidad pedagógica y tecnológica de Colombia, Tunja, Colombia. Actual Biol. 2012; 34 (97):241-252. 
17. Guo P. Yokoyama K, Piao F. Sakai K, Khalequzzaman M, Kamajima M, et al. Sick building syndrome by indoor air pollution in Dalian, China. Int. J. Environ. Res. Publich Health. 2013; 10:14891504.

18. Borges RCCO, Junior JCB, Oliveira FB, Brunherotti MA, Quemelo PRV. Avaliação da função pulmonar e sintomas respiratórios em trabalhadores da mineração de pirocloro. J Bras Pneumol. 2016; 42(4):279-285.

19. Pietrobon RC, Barbisan JN, Manfroi WC. Use of the fagerström test for nicotine dependence as an instrument to measure nicotine dependence. Rev HCPA. 2007; 27 (3):31-36

20. Vargas LS, Lucchese R, Silva AC, Benício PR, Vera I. Aplicação do teste de Fagerstrom: revisão integrativa. Rev Enfermagem UFPE on line. 2014; 9(2):731-44.

21. Santos JX. Mattos CR. O profissional bibliotecário e os desafios de um ambiente insalubre. Salvador. Monografia Instituto de Ciência da Informação. 2007.

22. Pantoja LDM, Neto JC, Nascimento RF, Nunes ABA. Percepção ocupacional: a qualidade do ar interno em biblioteca pública, Ceará, Brasil. Conex Ci e Tecnol. 2016; 10(3):118-124.

23. Souza RM, Andrade FM, Moura ABD. Teixeira PJZ. Sintomas respiratórios em trabalhadores de carvoarias nos municípios de Lindolfo Collor, Ivoti e Presidente Lucena, RS. J Bras Pneumol. 2010; 36(2):210-217.

24. Setto JM, Bonolo PF, Franceschini SCC. Relationship between health behaviors and self-reported diseases by public employees. Fisioter Mov. 2016; 29(3):477-485.

25. Ribeiro DRB. A Relação entre o uso de agrotóxicos e a saúde respiratória dos trabalhadores rurais. Alfenas: Universidade Federal de Alfenas. 2014

26. Freitas ALP, Souza RGB, Quintella HLMM. Qualidade de Vida no Trabalho do técnico-administrativo em IES públicas: uma análise exploratória. Rev Brasileira de Qualidade de Vida. 2013; 05(02):1-12.

27. Salazar PR. O uso do tabaco entre trabalhadores técnicos administrativos em educação de uma universidade pública do estado de minas gerais [dissertação). Juiz de Fora: Faculdade de Medicina, Universidade Federal de Juiz de Fora, Minas Gerais, Brasil. 2014

28. Echer IC, Corrêa APA, Lucena AF, Ferreira SAL, Knorst MM. Prevalência do tabagismo em funcionários de um hospital universitário. Rev Latino-Am Enfermagem. 2011; 19(1):1-8.

29. Ribeiro ALPC, Lubisco NML. Redução de fungos em ambiente de biblioteca: variabilidade de aplicação de neblina ativada. Rev Perspectivas em Gestão \& Conhecimento. 2016; 6(2):250-260. 